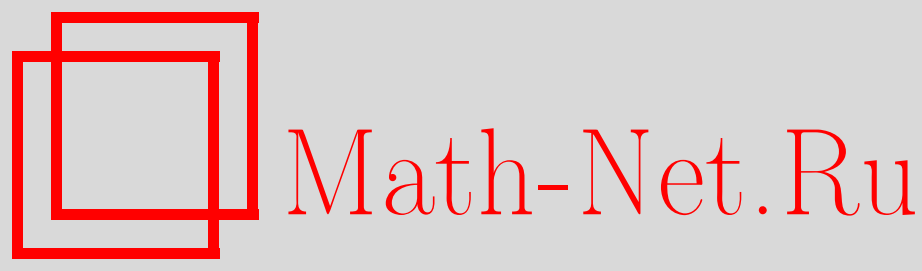

А. Ф. Крутов, М. А. Нефедов, В. Е. Троицкий, Аналитическое продолжение формфактора пиона из пространственноподобной во времениподобную область, ТМФ, 2013, том 174, номер 3, 383-397

DOI: https://doi.org/10.4213/tmf8406

Использование Общероссийского математического портала Math-Net.Ru подразумевает, что вы прочитали и согласны с пользовательским соглашением http://www . mathnet.ru/rus/agreement

Параметры загрузки:

IP : 54.172 .240 .79

26 апреля 2023 г., $16: 35: 52$

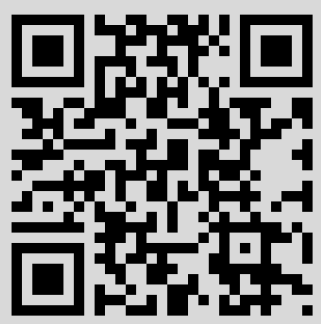




\title{
АНАЛИТИЧЕСКОЕ ПРОДОЛЖЕНИЕ ФОРМФАКТОРА ПИОНА ИЗ ПРОСТРАНСТВЕННОПОДОБНОЙ ВО ВРЕМЕНИПОДОБНУЮ ОБЛАСТЬ
}

\begin{abstract}
Решена задача аналитического продолжения электромагнитного формфактора пиона в комплексную плоскость переданных импульсов. Пион рассматривается как составная кварк-антикварковая система. Показано, что аналитические свойства формфактора, вычисленного в рамках релятивистской квантовой механики с прямым взаимодействием, соответствуют аналитическим свойствам, вытекающим из общих принципов квантовой теории поля.
\end{abstract}

Ключевые слова: формфактор, пион, аналитические свойства, релятивистская составная кварковая модель.

DOI: $10.4213 / \operatorname{tmf} 8406$

\section{1. ВВЕДЕНИЕ}

В релятивистской теории составных систем основными являются два следующих, существенно различающихся подхода. Один из них базируется на квантовой теории поля (КТП), а другой, предложенный в работе Дирака [1], основан на релятивистской квантовой механике (РКM) с прямым взаимодействием. Эти подходы отличаются один от другого прежде всего принципами, лежащими в их основе. Различными являются также области значений квадрата переданного импульса, в которых они имеют силу. К сожалению, эти подходы имеют столь фундаментальные различия, что ограничения одного подхода могут быть неприменимы в случае другого. Таким образом, сравнение этих двух методов весьма затруднительно. Подходы различны до такой степени, что могут вводить в заблуждение в результате того, что в них появляются совершенно разные по форме результаты. Более подробное обсуждение этих проблем можно найти в обзорах [2], [3].

* Самарский государственный университет, Самара, Россия.

E-mail: krutov@samsu.ru,nefedovma@gmail.com

${ }^{\dagger}$ Научно-исследовательский институт ядерной физики им. Д. В. Скобельцына, Московский государственный университет им. М. В. Ломоносова, Москва, Россия.

E-mail: troitsky@theory.sinp.msu.ru 
Основная цель настоящей работы - показать, что отсутствуют радикальные отличия в аналитических свойствах формфактора пиона, полученных в рамках РКМ и в КТП.

Все дальнейшее обсуждение мы будем проводить на примере формфактора пиона $F_{\pi}\left(Q^{2}\right)$ (здесь $Q^{2}$ - квадрат переданного импульса), рассматриваемого как составная система $q \bar{q}$. Пион представляет собой наиболее простой составной адронный объект, несмотря на это в настоящее время не существует теории, которая может полностью объяснить его поведение. Сложившаяся ситуация привлекает большое внимание как теоретиков, так и экспериментаторов.

Итак, первый подход - это метод теории поля. Основанный на принципах КТП, квантовой хромодинамики (КХД), он справедливо считается наиболее последовательным подходом к исследованию структуры адронов. С точки зрения вычисления $F_{\pi}\left(Q^{2}\right)$ наиболее надежно обоснованным методом является пертурбативная КХД. При больших $Q^{2}$ асимптотическое поведение формфактора пиона дается явной аналитической формулой [4]-[6]. Однако следует заметить, что результаты расчета $F_{\pi}\left(Q^{2}\right)$, опирающегося на пертурбативную КХД, лежат значительно ниже экспериментальных данных в доступной в настоящее время области значений $Q^{2}$ от нуля до 2.45 ГэВ $^{2}$ [7] (см. также приведенные в этой работе ссылки). Таким образом, данный метод никоим образом не может рассматриваться как способ количественного описания имеющихся экспериментальных данных.

Подход с использованием правила сумм КХД предназначен для интерполяции между пертурбативным и непертурбативным секторами с помощью дисперсионных методов в комбинации с операторным разложением. Так как практическая реализация подхода не может быть строго выведена из КХД, ее интуитивно понятная ценность состоит в том, что она обеспечивает некий мост между низкоэнергетическими свойствами других подходов и высокоэнергетическими свойствами КХД. В работах [8], [9] формфактор пиона был вычислен с использованием правила сумм КХД. K сожалению, результаты лежат примерно на $25 \%$ ниже существующих экспериментальных данных [7] и, что совсем неприятно, не обеспечивают правильного значения среднеквадратичного радиуса пиона, который весьма надежно определен из прямых экспериментов по рассеянию пионов на электронах атомной оболочки [10].

Описание формфакторов именно составной системы методами КХД налагает некоторые дополнительные ограничения (см., например, монографию [11]). Дело в том, что составной системе должен соответствовать полюс, а в рамках теории поля это невозможно обеспечить каким-либо конечным набором диаграмм. Обычно (см. обзор [12]) в основу описания составных частиц кладут формализм Бете-Солпитера [13], [14] или квазипотенциальный подход [15]. В частности, формфактор пиона исследован в работе [16] в рамках квазипотенциального метода.

В непертурбативных подходах используются также дисперсионные соотношения, которые основаны на ограничениях, налагаемых причинностью и аналитичностью. Эти соотношения связывают времениподобную и пространственноподобную области формфактора пиона в комплексной плоскости. В принципе, это точная техника, но в силу нашего неполного знания амплитуды рассеяния во всей комплексной плоскости и, в частности, неполного знания всех полюсов во времениподобной области появляется полоса неопределенностей. Существует достаточно много работ, например [17]-[22], посвященных исследованию этого вопроса. Результаты некоторых из них противоречат друг другу. Исходным моментом является большое количество 
экспериментальных данных по процессу $e^{+} e^{-} \rightarrow \pi^{+} \pi^{-}$. Но, как известно из теории аналитических функций, знание, пусть даже большого количества отдельных точек, не позволяет построить однозначное аналитическое продолжение, что приводит к достаточно большому разбросу результатов в пространственноподобной области полосе неопределенности.

Наконец, отметим, что некоторые авторы применяют к формфактору пиона голографическую модель КХД [23]-[26]. К сожалению, эта интересная модель сильно зависит от выбора параметров и не обеспечивает точного описания формфактора пиона [7].

Второй подход базируется на предложенном Дираком [1] методе PKM с прямым взаимодействием. Концепция прямого взаимодействия и характер теории, которая строится на основе этой концепции, подробно изложены в работе [27]. Прямое взаимодействие физически означает взаимодействие на расстоянии, которое передается без посредства полей или других носителей, имеющих собственные степени свободы. Отсутствие посредников, передающих взаимодействие между частицами, математически проявляется в том, что потенциал взаимодействия (или, в квантовом случае, оператор взаимодействия) выражается полностью через переменные, описывающие степени свободы частиц.

Развитие этого подхода и ссылки можно найти в ряде обзоров [2], [28]-[30]. Наиболее прямой метод включения взаимодействия в точную модель РКМ был предложен в работе [31]. Обобщение этого метода и более поздние ссылки даны в статье [32]. В настоящее время для описания составных систем успешно используется вариант PKM, восходящий по духу к работе [33] и использующий двойные интегралы дисперсионного типа по полной массе составных систем. Эти интегралы возникают в результате решений уравнений типа Мусхелишвили-Омнеса. Метод был, в частности, успешно применен к расчету формфактора пиона [34], причем продемонстрировал свою предсказательную силу (см. подробности в работе [35]). Мы используем этот метод в качестве отправной точки в настоящей работе для описания аналитических свойств формфактора пиона и продолжения его во времениподобную область.

Как было отмечено выше, одной из основных нерешенных теоретических проблем РКМ является проблема ее связи с фундаментальными принципами КТП. В настоящее время не существует ответа на вопрос, можем ли мы, исходя из принципов КТП, вывести исходные аксиомы РКМ. Настоящая работа отчасти связана с этой проблемой. Мы ставим своей задачей сравнение следствий из принципов КТП и модельно независимых следствий из аксиом РКМ. Так, в нашей работе [36] было показано, что при больших пространственноподобных переданных импульсах в пределе нулевых масс конституентов асимптотическое поведение формфактора пиона в мгновенной форме РКМ совпадает с предсказаниями кваркового счета и КХД. В настоящей работе мы сравним предсказания аналитических свойств формфактора пиона в комплексной плоскости переданных импульсов, которые следуют из общих принципов КТП, и аналитические свойства формфактора, полученного путем аналитического продолжения его интегрального представления в пространственноподобной области, выведенного в мгновенной форме PKM [37]. Отметим, что задача аналитического продолжения формфактора в рамках РКМ поставлена нами впервые. В настоящей работе для описания аналитических свойств формфактора пиона и его поведения при времениподобных переданных импульсах используется пуанкаре-инвариантная составная кварковая модель, упомянутая выше. 
Одной из особенностей нашего подхода является использование метода релятивистски-инвариантной параметризации матричных элементов локальных операторов [38], который позволил решить важную для всех вариантов РКМ проблему построения оператора сохраняющегося лоренц-ковариантного электромагнитного тока для составных систем [39].

В настоящей работе в рамках пуанкаре-инвариантной составной кварковой модели осуществлено аналитическое продолжение электромагнитного формфактора пиона из области пространственноподобных переданных импульсов в комплексную плоскость. Показано, что аналитические свойства формфактора фактически совпадают со свойствами, которые предсказываются в рамках подхода КТП. Обнаружено, что аналитическое продолжение сильно зависит от вида волновой функции кварков в пионе. Получены ограничения на волновые функции, вытекающие из условия правильного резонансного поведения формфактора пиона в области времениподобных переданных импульсов. Построена простейшая реалистичная волновая функция, дающая стандартное резонансное поведение формфактора в этой области значений переданных импульсов.

\section{2. ФОРМФАКТОР ПИОНА В МГНОВЕННОЙ ФОРМЕ РКМ В ПРОСТРАНСТВЕННОПОДОБНОЙ ОБЛАСТИ}

Выражения для электромагнитных формфакторов двухчастичной системы в рамках мгновенной формы PKM в так называемом модифицированном импульсном приближении были получены в работах [37], [39]. В приближении точечных конституентных кварков выражение для электрического формфактора пиона в пространственноподобной области может быть приведено к виду

$$
F_{\pi}(t)=\frac{1}{4} \int_{\Omega_{s s^{\prime}}} \frac{\varphi(s) \varphi\left(s^{\prime}\right)}{\sqrt{\left(s-4 M^{2}\right)\left(s^{\prime}-4 M^{2}\right)}} \frac{-t\left(s+s^{\prime}-t\right)}{\lambda^{3 / 2}\left(s, t, s^{\prime}\right)} d s d s^{\prime},
$$

где $M$ - масса конституента, $\lambda\left(s, t, s^{\prime}\right)=s^{2}+t^{2}+\left(s^{\prime}\right)^{2}-2\left(s s^{\prime}+s t+s^{\prime} t\right)$. При $t<0$ данное выражение представляет собой двойной интеграл от вещественной функции, область интегрирования имеет вид

$$
\Omega_{s s^{\prime}}=\left\{\left(s, s^{\prime}\right) \in \mathbb{R}^{2}: s \in\left[4 M^{2},+\infty\right), s^{\prime} \in\left[s_{1}(s, t), s_{2}(s, t)\right]\right\},
$$

а пределы интегрирования таковы:

$$
s_{1,2}(s, t)=s+t-\frac{t s}{2 M^{2}} \mp \frac{1}{2 M^{2}} \sqrt{-t\left(4 M^{2}-t\right) s\left(s-4 M^{2}\right)} .
$$

Волновая функция $\varphi(s)$ в мгновенной форме РКМ связана с волновой функцией $u(k)$, зависящей от модуля относительного трехмерного импульса конституента:

$$
\varphi(s)=\sqrt[4]{s} k u(k), \quad s=4\left(k^{2}+M^{2}\right) .
$$

Волновая функция нормирована условием

$$
\int_{-\infty}^{\infty} k^{2} u^{2}(k) d k=1
$$

Формулы (1)-(3) были с успехом применены в работах [34], [35], [37] для описания электромагнитного формфактора пиона при всех достигнутых в экспериментах на сегодняшний день пространственноподобных переданных импульсах. Заметим, что данное выражение в пределе нулевой массы конституентов дает асимптотику 
формфактора при больших переданных импульсах, не зависящую от вида волновой функции кварков в пионе [36] и совпадающую с предсказаниями кваркового счета [40], [41], которые справедливы в кварк-партонной модели [40], [41] и КХД [42].

$\mathrm{C}$ технической точки зрения задача построения формфактора пиона в комплексной плоскости переданных импульсов сводится к аналитическому продолжению выражения (1) с отрицательной части вещественной оси в комплексную плоскость параметра $t$.

Можно показать, что свойства аналитического продолжения выражения (1) сильно зависят от типа волновой функции конституентов. Таким образом, возникает задача выяснения условий, которые требуется наложить на волновые функции, чтобы получить, например, экспериментально установленное резонансное поведение формфактора во времениподобной области переданных импульсов, т. е. на положительной оси параметра $t$.

\section{3. АНАЛИТИЧЕСКОЕ ПРОДОЛЖЕНИЕ ФОРМФАКТОРА ПИОНА В КОМПЛЕКСНУЮ ПЛОСКОСТЬ ПЕРЕДАННЫХ ИМПУЛЬСОВ}

Из условия микропричинности в КТП следует, что формфактор пиона является аналитической функцией в комплексной плоскости параметра $t$ с разрезом, идущим от $4 m_{\pi}^{2}$ до $+\infty$, где $m_{\pi}$ - масса пиона (см., например, работы [21], [22] и ссылки, приведенные в них). В настоящем разделе мы покажем, что аналогичные свойства имеют место и в нашей формулировке составной кварковой модели. Как мы уже отмечали, этот факт является весьма интересным с точки зрения прояснения связи между фундаментальным подходом КТП и феноменологическими составными кварковыми моделями.

Аналитическое продолжение выражения (1) из области пространственноподобных переданных импульсов $(t<0)$ в комплексную плоскость параметра $t$ путем подстановки комплексных значений в двойной интеграл провести не удается. Причина заключается в том, что переменная $s^{\prime}$ в этом случае становится комплексной, и интеграл по вещественной переменной превращается в интеграл по некоторому пути в комплексной плоскости. При $\operatorname{Re} t>0$ двойной интеграл расходится, когда концевые точки $s_{1,2}$ пути интегрирования в комплексной плоскости переменной $s^{\prime}$, заданные в (2), совпадают с особенностями подынтегрального выражения. Данное совпадение не удается устранить ни путем деформации контура в плоскости $s^{\prime}$, ни путем выхода в комплексную плоскость переменной $s$. Эту трудность можно обойти с помощью некоторой нелинейной замены переменных в двойном интеграле (1).

Заметим, что подынтегральное выражение в (1) симметрично относительно перестановки переменных $s$ и $s^{\prime}$, и осью симметрии области интегрирования $\Omega_{s s^{\prime}}$ является прямая $s^{\prime}=s$. Следовательно, мы можем интегрировать по половине области $\Omega_{s s^{\prime}}$, т. е. по области

$$
\widetilde{\Omega}_{s s^{\prime}}=\left\{\left(s, s^{\prime}\right) \in \mathbb{R}^{2}: s \in\left[4 M^{2}, \infty\right), s^{\prime} \in\left[s_{1}(s, t), s\right]\right\} .
$$

Выполним в интеграле (1) нелинейную замену переменных $x=s+s^{\prime}-t, y=\sqrt{4 s s^{\prime}}$. Область $\widetilde{\Omega}_{s s^{\prime}}$ при такой замене переходит в область

$$
\Omega_{x y}=\left\{(x, y) \in \mathbb{R}^{2}: x \in\left[x_{\min }(t),+\infty\right), y \in\left[y_{1}(x, t), y_{2}(x, t)\right]\right\},
$$


где

$$
\begin{gathered}
x_{\min }(t)=4 M^{2}-t+2 M \sqrt{4 M^{2}-t}, \\
y_{1}(x, t)=x \sqrt{\frac{4 M^{2}}{4 M^{2}-t}}, \quad y_{2}(x, t)=x+t .
\end{gathered}
$$

Выражение (1) в новых переменных принимает вид

$$
F_{\pi}(t)=\frac{-t}{8 \sqrt{2}} \int_{x_{\min }(t)}^{+\infty} \int_{y_{1}(x, t)}^{y_{2}(x, t)} U(x, y, t) \frac{x y^{3 / 2}}{\left(x^{2}-y^{2}\right)^{3 / 2} \sqrt{y_{2}^{2}(x, t)-y^{2}}} d y d x .
$$

Здесь функция $U(x, y, t)$ представляет собой произведение функций $u(k)$ в новых переменных:

$$
U\left(s+s^{\prime}-t, \sqrt{4 s s^{\prime}}, t\right)=u(k) u\left(k^{\prime}\right) .
$$

Выражение (6) в комплексной плоскости параметра $t$ представляет собой двойной интеграл по некоторым путям в комплексных плоскостях переменных $x$ и $y$. Обсудим выбор путей интегрирования. При вещественных отрицательных $t$ (при пространственноподобных переданных импульсах) начальные и конечные точки интегрирования принадлежат вещественным осям. Тогда мы выбираем пути интегрирования лежащими на вещественных осях, при этом пути не пересекают особенностей подынтегральной функции. Такое интегрирование дает значения формфактора пиона в пространственноподобной области переданных импульсов, совпадающие с исходным интегралом (1). При комплексных $t$ начальные и конечные точки выходят в комплексную плоскость, и пути интегрирования необходимо выбирать специальным образом. Выбор путей мы обсудим ниже при доказательстве следующей теоремы, в которой формулируются достаточные условия того, что выражение (6) при условии, что функция $U(x, y, t)$ выбрана из некоторого класса, и при некотором выборе путей интегрирования определяет функцию, аналитическую в плоскости параметра $t$ с разрезом.

Теорема 1. Интегральное представление (6), рассматриваемое как двойной интеграл по комплексным переменным $x$ и y, определяет функиию, аналитическую в плоскости параметра $t$ с разрезом вдоль действительной оси от $4 M^{2}$ до $+\infty$, , ри выполнении следующих условий:

1) путь интегрирования $\gamma_{x}$ в комплексной плоскости переменной $x$ начинается в точке $x_{\min }(t)$ и уходит на бесконечность вдоль положительного направления вещественной оси, оставаясь в полуплоскости $\operatorname{Re} x>0$;

2) функция $U(x, y, t)$ является аналитической по переменной у и может иметь в этой плоскости конечное число полюсов и точек ветвления, причем любая особая точка $y_{\mathrm{s}}$ не совпадает с $y_{1,2}(x, t)$ и при $|x| \rightarrow \infty$ величина $\left|y_{\mathrm{s}}\right|$ растет не бъстрее чем $|x|$;

3) для всех $t>4 M^{2}$ справедливо равенство $U\left(x^{*}, y^{*}, t-i 0\right)=U^{*}(x, y, t+i 0)$.

ДокАЗАТЕЛЬство. Обсудим сначала пути интегрирования в выражении (6). Обратим внимание, что начальная точка пути интегрирования $\gamma_{x}$ в плоскости переменной $x$ непрерывным образом зависит от параметра $t$, значения которого лежат в комплексной плоскости с разрезом вдоль вещественной оси от $4 M^{2}$ до $+\infty$. В силу аналитичности функции $U(x, y, t)$ и вследствие равенства $(7)$ положение особенностей подынтегральной функции в комплексной плоскости переменной $y$ непрерывным образом зависит от $x$ и параметра $t$, который лежит в комплексной плоскости 
с разрезом. Заметим, что зависимость начальной и конечной точек $y_{1,2}(x, t)$ от переменной $x$ и от параметра $t$ из комплексной плоскости с разрезом также является непрерывной.

Таким образом, путь $\gamma_{y}$, соединяющий точки $y_{1,2}(x, t)$, можно выбрать так, чтобы он не пересекал особенности и разрезы подынтегрального выражения в плоскости переменной $y$. В силу непрерывности рассматриваемых функций существует семейство гомотопных путей, соединяющих указанные точки и не пересекающих особенности и разрезы. Вследствие аналитичности подынтегрального выражения и гомотопности путей из данного семейства значение интеграла (6) определяется только положением начальных и конечной точек интегрирования в плоскостях переменных $x$ и $y$. Положение этих точек непрерывным образом зависит от $t$, что приводит к непрерывности данного интегрального представления в плоскости параметра $t$ с описанным выше разрезом.

Из определения (7) функции $U(x, y, t)$ и условия (4), справедливого при вещественных значениях аргументов, следует оценка при $|x| \rightarrow \infty$ :

$$
\max _{y \in \gamma_{y}}|U(x, y, t)| \sim o\left(|x|^{-1 / 2}\right) .
$$

В силу этого для всех $x \in \gamma_{x}$ на указанном семействе путей выполняется неравенство

$$
\max _{y \in \gamma_{y}}|U(x, y, t)|<\infty .
$$

Докажем равномерную сходимость интегрального представления для формфактора (6) в плоскости параметра $t$. Для этого достаточно доказать абсолютную сходимость интеграла при условиях теоремы. Для дальнейших оценок деформируем контур в плоскости переменной $у$ так, чтобы он огибал особенности подынтегральной функции. Произведем параметризацию контура интегрирования следующим образом:

$$
y(x, t, \tau)=y_{2}(x, t)-\left(y_{2}(x, t)-y_{1}(x, t)\right) f(\tau), \quad \tau \in[0,1],
$$

где $f(\tau)$ - гладкая комплекснозначная функция вещественного аргумента, $f(1)=1$, $f(0)=0$.

Рассмотрим интеграл от модуля подынтегрального выражения в (6):

$$
I(t)=\int_{x_{\min }(t)}^{+\infty} \int_{y_{1}(x, t)}^{y_{2}(x, t)}|U(x, y, t)| \frac{|x||y|^{3 / 2}}{\left|\left(x^{2}-y^{2}\right)^{3 / 2} \sqrt{y_{2}(x, t)^{2}-y^{2}}\right|}|d y||d x| .
$$

Отдельно рассмотрим внутренний интеграл по комплексной переменной $y$. Для этого интеграла с учетом условия (9) и параметризации (10) справедлива следующая оценка:

$$
\begin{aligned}
& \int_{y_{1}(x, t)}^{y_{2}(x, t)}|U(x, y, t)| \frac{|y|^{3 / 2}|d y|}{\left|\left(x^{2}-y^{2}\right)^{3 / 2} \sqrt{y_{2}^{2}(x, t)-y^{2}}\right|}|d y| \leqslant \\
& \quad \leqslant \max _{y \in \gamma_{y}}|U(x, y, t)| \cdot M(x, t) \cdot\left|\sqrt{y_{2}(x, t)-y_{1}(x, t)}\right| \int_{0}^{1} \frac{\left|f^{\prime}(\tau)\right|}{\sqrt{|f(\tau)|}} d \tau,
\end{aligned}
$$

где

$$
M(x, t)=\max _{\tau \in[0,1]} \frac{|y(x, t, \tau)|^{3 / 2}}{\left|x^{2}-y^{2}(x, t, \tau)\right|^{3 / 2}\left|\sqrt{y_{2}(x, t)+y(x, t, \tau)}\right|} .
$$


Из условий, налагаемых на контур в плоскости $y$, следует, что $|M(x, t)|<\infty$. Всегда можно выбрать форму контура и его параметризацию так, чтобы выполнялось условие $|f(\tau)| \geqslant \tau$. Следовательно, справедлива следующая оценка:

$$
\int_{0}^{1} \frac{\left|f^{\prime}(\tau)\right|}{\sqrt{|f(\tau)|}} d \tau \leqslant \max _{\tau \in[0,1]}\left|f^{\prime}(\tau)\right| \int_{0}^{1} \frac{d \tau}{\sqrt{\tau}}=2 \max _{\tau \in[0,1]}\left|f^{\prime}(\tau)\right| .
$$

Принимая во внимание сделанные оценки, для исходного интеграла получаем

$$
I(t) \leqslant 2 \int_{x_{\min }(t)}^{\infty} \max _{\tau \in[0,1]}\left|f^{\prime}(\tau)\right||x|\left|\sqrt{y_{2}(x, t)-y_{1}(x, t)}\right| M(x, t) \max _{y \in \gamma_{y}}|U(x, y, t)||d x| .
$$

Подынтегральное выражение в правой части конечно при всех $x \in \gamma_{x}$, поэтому для сходимости интеграла достаточно, чтобы оно убывало быстрее чем $x^{-1}$. Асимптотическую оценку подынтегрального выражения при $|x| \rightarrow \infty$ можно провести, учитывая, что $y_{1,2}(x, t) \sim x$ и $y(x, t, \tau) \sim x$. Величина $\max _{\tau \in[0,1]}\left|f^{\prime}(\tau)\right|$ является конечной в силу условия 2 теоремы и непрерывности производной на отрезке. Принимая во внимание эти оценки, можно показать, что при $|x| \rightarrow \infty$ подынтегральное выражение в (12) ведет себя как $|x|^{-1 / 2} \max _{y \in \gamma_{y}}|U(x, y, t)|$. Отсюда с учетом условия (8) следует, что данный интеграл сходится. Таким образом, мы показали абсолютную сходимость интеграла (11) и, следовательно, равномерную сходимость интеграла (6). Из равномерной сходимости интеграла и голоморфности подынтегральной функции по переменным $x$ и $y$ в окрестности путей интегрирования следует аналитичность функции (6) в плоскости параметра $t$.

Наличие разреза в плоскости параметра $t$ легко проверяется на основе свойств пределов интегрирования в выражении (6). При $t<4 M^{2}$ пределы интегрирования (5) действительны, и выражение (6) принимает действительные значения. При $t=\tau \pm i 0$ (для $\tau>4 M^{2}$ ) пределы интегрирования становятся комплексными, причем они принимают комплексно-сопряженные значения на верхнем и нижнем берегах разреза, идущего вдоль вещественной оси от $4 M^{2}$ до $+\infty$. Это приводит к тому, что функция (6) претерпевает разрыв на данном разрезе, и при выполнении условия 4 теоремы значения формфактора на берегах разреза связаны условием $F_{\pi}(\tau+i 0)=F_{\pi}^{*}(\tau-i 0)$. Таким образом, теорема доказана.

Полученные нами аналитические свойства формфактора отличаются от аналитических свойств, получающихся в рамках подхода КТП, только положением точки ветвления на вещественной оси: эта точка есть $4 M^{2}$ и $4 m_{\pi}^{2}$ соответственно.

\section{4. ОПИСАНИЕ РЕЗОНАНСНОГО ПОВЕДЕНИЯ ФОРМФАКТОРА ПИОНА ВО ВРЕМЕНИПОДОБНОЙ ОБЛАСТИ ПЕРЕДАННЫХ ИМПУЛЬСОВ}

Установленные в предыдущем разделе аналитические свойства формфактора пиона не зависят от явного вида волновых функций в выражениях (1)-(4). Однако детали поведения формфактора в комплексной плоскости переданных импульсов существенно зависят от формы волновой функции. Основной качественной особенностью поведения формфактора в комплексной плоскости, установленной экспериментально, является наличие на положительной части вещественной оси - во времениподобной области - двух близколежащих резонансов, которые соответствуют $\rho$ и $\omega$-мезонам. Поэтому задача построения формфактора в комплексной плоскости 
переданных импульсов не может считаться решенной, если аналитическое продолжение формфактора не имеет резонанса на положительной части вещественной оси.

Можно показать, что волновые функции в импульсном представлении, не имеющие полюсов в комплексной плоскости импульсов, не дают резонансного поведения формфактора пиона при времениподобных переданных импульсах. В частности, это касается широко используемой в составных моделях волновой функции гауссовского типа (см., например, работы [43]-[46], причем этот результат не зависит от значений параметров модели. Таким образом, возникает задача определения вида волновых функций, приводящих к резонансам, и установления связи положения резонанса в формфакторе с положением полюсов волновой функции. Основной идеей при решении этой задачи является введение таких имеющих полюсы волновых функций, которые приводят к появлению полюсов у формфактора (6) на нефизическом листе. Как мы покажем ниже, это дает нужное резонансное поведение формфактора на положительной части вещественной оси.

Для удобства введем новую переменную

$$
t=4 M^{2}-z^{2},
$$

формфактор как функция этой переменной становится однолистной функцией. При такой замене пространственноподобная область $t<0$ переданных импульсов отображается на положительную часть действительной оси переменной $z$; берега разреза в плоскости параметра $t$, начинающегося от $4 M^{2}$ и идущего вдоль положительной части вещественной оси, отображаются на мнимую ось; физический лист формфактора пиона отображается на правую полуплоскость, а нефизический лист - на область $\operatorname{Re} z<0$. Мы считаем, что аргумент комплексной переменной $z$ изменяется в пределах $-\pi<\arg z<\pi$. В плоскости переменной $z$ функция (6) является однозначной в силу того, что однозначными становятся выражения для пределов интегрирования:

$$
x_{\min }(z)=z^{2}+2 M z, \quad y_{1}(x, z)=\frac{2 M}{z} x, \quad y_{2}(x, z)=x+4 M^{2}-z^{2} .
$$

Пусть максимальное значение формфактора на положительной части вещественной оси достигается в точке $t_{\mathrm{r}}>4 M^{2}$. При характерных массах конституентов в составных кварковых моделях и известном положении резонанса это условие может быть выполнено. Введем в волновую функцию полюс, который приведет к наличию у формфактора особенности на нефизическом листе, и покажем, что это влечет резонансное поведение формфактора во времениподобной области. Пусть резонансу в окрестности точки $t_{\mathrm{r}}$ соответствует полюс формфактора, расположенный в точке

$$
z_{\mathrm{s}}=z_{\mathrm{s}}^{\prime \prime}+i z_{\mathrm{s}}^{\prime}=z_{\mathrm{s}}^{\prime \prime}+i \sqrt{t_{\mathrm{r}}-4 M^{2}}, \quad z_{\mathrm{s}}^{\prime \prime}<0,
$$

в нефизической области переменной z. В силу действительности формфактора в пространственноподобной области картина особенностей в плоскости переменной $z$ должна быть симметричной относительно действительной оси. Наличие полюса функции $F_{\pi}(z)$ в точке $z_{\mathrm{s}}$ означает, что интеграл (6) расходится в этой точке.

Расходимость интеграла мы можем обеспечить, манипулируя особенностями функции $U(x, y, t)$. Выберем точку в плоскости $y$, в которую нужно поместить особенность функции $U(x, y, t)$, так, чтобы интеграл расходился в заданной точке $z_{\mathrm{s}}$. Эта особенность должна принадлежать всем возможным контурам в плоскости переменной $y$, т. е. это должна быть концевая точка контура. Однако, как видно из формул 
для пределов интегрирования, положение концевых точек $y_{1}$ и $y_{2}$ зависит от значения второй переменной интегрирования $x$. Существует единственная точка $x$, через которую проходят все контуры интегрирования в плоскости переменной $x,-$ конечная точка $x_{\min }$.

Существует единственная точка в плоскости переменной $y$, не зависящая от формы контуров: точка, в которой находятся концы контура $y_{1}$ и $y_{2}$, когда $x=x_{\min }$. Именно в эту точку мы поместим особенность функции $U(x, y, t)$. Таким образом, положение полюса подынтегральной функции в плоскости переменной $y$ определяется значением пределов интегрирования:

$$
y_{\mathrm{s}}=y_{1}\left(x_{\min }\left(4 M^{2}-z_{\mathrm{s}}^{2}\right), 4 M^{2}-z_{\mathrm{s}}^{2}\right)=y_{2}\left(x_{\min }\left(4 M^{2}-z_{\mathrm{s}}^{2}\right), 4 M^{2}-z_{\mathrm{s}}^{2}\right)=2 M\left(z_{\mathrm{s}}+2 M\right) .
$$

Таким образом, волновые функции, приводящие к резонансному поведению формфактора пиона при времениподобных переданных импульсах, должны удовлетворять следующим условиям:

1) волновая функция $u(k)(3)$ имеет полюс в точке $k_{\mathrm{s}}^{2}=M\left(z_{\mathrm{s}}-2 M\right) / 4$;

2) будучи фурье-образом радиальной части волновой функции в координатном представлении, функция $u(k)$ является четной;

3) волновая функция $u(k)$ является действительной при действительных $k$.

Введение в подынтегральную функцию особенности в соответствии с условием 1 не приводит к трудностям при вычислении интегралов (6) при значениях квадрата переданных импульсов $t<4 M^{2}$. Однако ситуация меняется при $t>4 M^{2}$. Как видно из выражений (5) для пределов интегрирования, при изменении $x$ пределы интегрирования движутся в комплексной плоскости переменной $y$. При этом контур интегрирования может пересечь введенную нами особенность, что приведет к выходу особенности формфактора на физический лист. Таким образом, требуется задание правил обхода введенной нами особенности в плоскости переменной $y$.

Для задания правил обхода наложим естественное условие непрерывности формфактора как функции квадрата переданного импульса в точке $4 M^{2}$ или, в терминах переменной $z(13)$, в точке $z=0$. Из выражений (14) для пределов интегрирования следует, что $y_{1} \rightarrow \infty$ при $z \rightarrow 0$. Для того чтобы предел в точке $z=0$ не зависел от пути, по которому мы к ней приближаемся, необходимо, чтобы пути интегрирования в плоскости $y$, соединяющие точки $y_{1}$ и $y_{2}$, были гомотопны друг другу при всех $x \in \gamma_{x}$. Направление обхода контура следует выбрать из требования непрерывного сшивания с формфактором при $t<0$.

Простейшей рациональной функцией, удовлетворяющей всем этим условиям, является функция

$$
u_{\mathrm{M}}(k)=N\left[\left(k^{2}-k_{\mathrm{s}}^{2}\right)\left(k^{2}-\left(k_{\mathrm{s}}^{*}\right)^{2}\right)\right]^{-n} .
$$

Соответствующая функция $U(x, y, t)$ из $(7)$ имеет вид

$$
U_{\mathrm{M}}(x, y, t)=N^{2}(16)^{2 n}\left[\left(\frac{y^{2}}{4}+\alpha(x+t)+\alpha^{2}\right) \cdot\left(\alpha \rightarrow \alpha^{*}\right)\right]^{-n},
$$

где $\alpha=-4\left(k_{\mathrm{s}}^{2}+M^{2}\right)$. Нормировочная постоянная $N$ определяется из условия нормировки (4). Явный ее вид мы не приводим из-за громоздкости выражения, но используем при численном интегрировании. Таким образом, полученное аналитическое продолжение с волновыми функциями вида (16) содержит следующие параметры: $t_{\mathrm{r}}$ и $z^{\prime \prime}$ из (15), масса конституента $M$ и степень $n$ волновой функции. Эти параметры могут быть фиксированы путем подгонки экспериментальных данных 


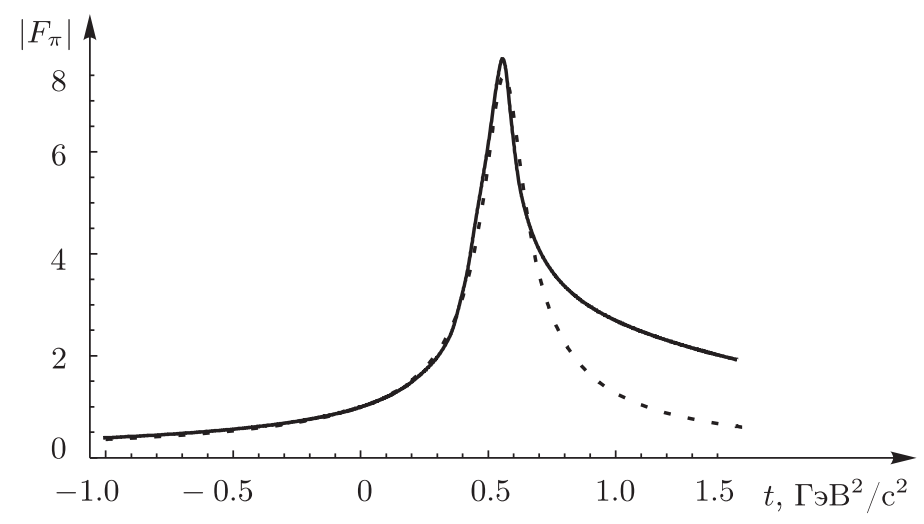

Рис. 1. Функция $\left|F_{\pi}(t)\right|$ : сплошная кривая отвечает выражению (6), штриховая кривая - аппроксимации Брейта-Вигнера.

по параметрам $\rho$-мезонного резонанса в формфакторе пиона, а именно его высоте, ширине и положению.

Для описания экспериментальных данных по формфактору пиона мы использовали фитирование (см. работу [25]) при помощи известной формулы Брейта-Вигнера

$$
F_{\pi \mathrm{BW}}(t)=\frac{4 \kappa^{2}}{4 \kappa^{2}-t-2 i \kappa \Gamma}
$$

где $\kappa=0.375$ ГэВ, $\Gamma=0.1$ ГэВ (параметры взяты из работы [25]). Параметры нашей модели подбирались численно из соображений наилучшего согласия значений модуля формфактора с аппроксимацией экспериментальных данных по Брейту-Вигнеру: мы получили

$$
t_{\mathrm{r}}=0.57 \Gamma \ni \mathrm{B}^{2}, \quad z_{\mathrm{s}}^{\prime \prime}=-0.01, \quad M=0.32 \Gamma \ni \mathrm{B}, \quad n=0.86 .
$$

Заметим, что параметр $t_{\mathrm{r}}$ имеет смысл массы резонанса, и его значение очень близко к значению 0.5625 Гэ ${ }^{2}$, задаваемому формулой (17), а значение массы конституентного кварка является типичным для различных формулировок составной кварковой модели, в которых массы конституентов лежат в интервале $(0.2-0.33)$ ГэВ (см., например, [34], [43]-[47]). Параметр $z_{\mathrm{s}}^{\prime \prime}$, определяющий положение особенности формфактора на нефизическом листе, и параметр $n$ волновой функции носят явно модельный характер и являются чисто подгоночными.

Результаты численного интегрирования выражения (6) с волновой функцией (16) и расчеты по формуле Брейта-Вигнера представлены на рис. 1. Как видно из рисунка, аналитическое продолжение формфактора пиона (6) из пространственноподобной области в комплексную плоскость, проведенное в настоящей работе, при простейшем выборе волновой функции в виде (16) дает довольно хорошее качественное описание формфактора во времениподобной области. Отклонение от формулы Брейта-Вигнера или, что то же самое, от эксперимента в области значений квадрата переданного импульса, лежащих выше точки максимума, объясняется, по-видимому, недостаточной реалистичностью выбора волновой функции в виде (16). Заметим, 


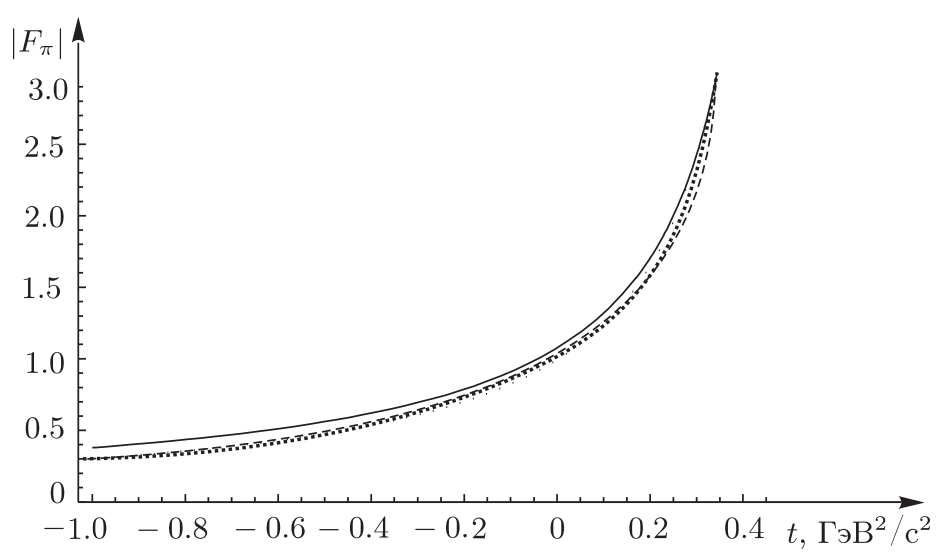

Рис. 2. Сравнение расчета формфактора пиона с известными дисперсионными соотношениями: точки отвечают непосредственному вычислению по формуле (6), сплошная кривая - дисперсионному соотношению (19), штриховая кривая - дисперсионному соотношению (20). Результаты расчета по формуле (6) фактически совпадают с результатами расчетов по дисперсионной формуле (20).

что выбор волновой функции слабо влияет на описание формфактора в пространственноподобной области, таким образом, для прецизионного описания формфактора пиона во времениподобной области переданных импульсов необходимо более сложное выражение для волновой функции, например с бо́льшим числом полюсов.

Отметим следущие обстоятельства. Во-первых, несмотря на простоту, волновая функция (16) отвечает общепринятым представлениям об описании взаимодействия в составной модели. Можно показать, что она является решением динамического уравнения РКМ с потенциалом межкваркового взаимодействия, имеющим кулоновское поведение на малых расстояниях и конфайнмент, который на промежуточных расстояниях является линейным, а при увеличении расстояния переходит в нелинейный. Во-вторых, для прецизионных расчетов может быть использована обобщенная формула типа Брейта-Вигнера, но, конечно, с дополнительными параметрами [48].

\section{5. ПРОВЕРКА КОРРЕКТНОСТИ АНАЛИТИЧЕСКОГО ПРОДОЛЖЕНИЯ}

Корректность построенного нами аналитического продолжения может быть продемонстрирована следующим образом. Сначала мы проверим, насколько точно удовлетворяет построенный нами формфактор в пространственноподобной области известному дисперсионному соотношению

$$
F_{\pi}(t)=\frac{1}{\pi} \int_{4 M^{2}}^{\infty} \frac{\operatorname{Im} F_{\pi}\left(t^{\prime}\right)}{t^{\prime}-t} d t^{\prime},
$$

а также дисперсионному соотношению с одним вычитанием (с ослабленным требованием к убыванию формфактора на бесконечности)

$$
F_{\pi}(t)=1+\frac{t}{\pi} \int_{4 M^{2}}^{\infty} \frac{\operatorname{Im} F_{\pi}\left(t^{\prime}\right)}{t^{\prime}\left(t^{\prime}-t\right)} d t^{\prime} .
$$




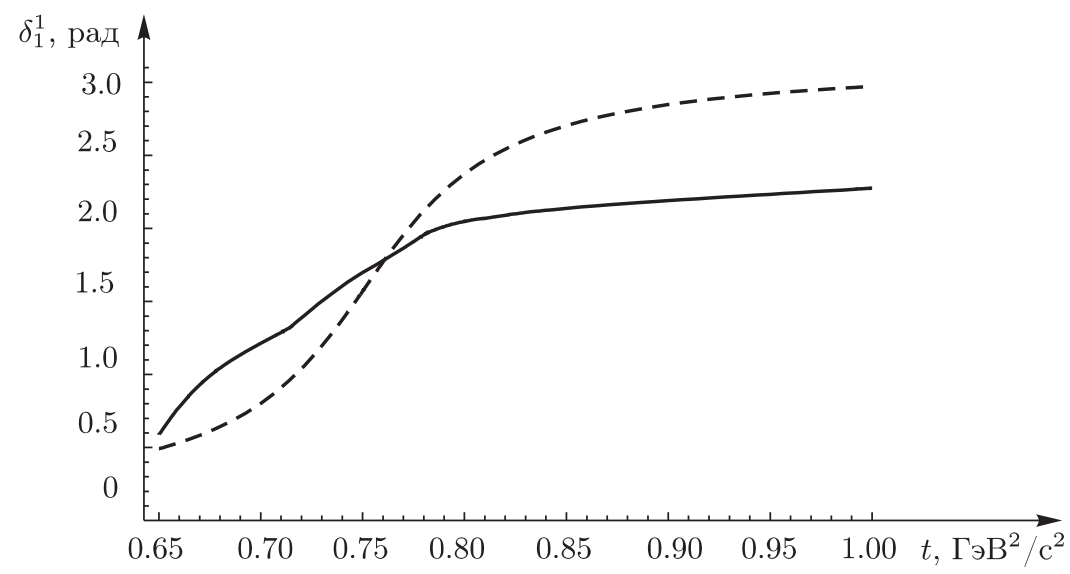

Рис. 3. Фаза $\pi \pi$-рассеяния: штриховой линией изображена фаза, отвечающая формуле Брейта-Вигнера, сплошной линией - результат расчета фазы по формуле (6).

Сравнение нашего результата с формулами (19), (20) приведено на рис. 2. Для расчетов использовались значения параметров (18). Видно, что построенный нами в комплексной плоскости формфактор пиона удовлетворяет дисперсионным соотношениям с хорошей точностью.

Мы также проверили корректность предложенного аналитического продолжения на основе условия унитарности $S$-матрицы $\pi \pi$-рассеяния, которое в терминах упругого формфактора пиона записывается в виде (см., например, книгу [49])

$$
\operatorname{Im} F_{\pi}(t)=F_{\pi}(t) e^{-i \delta_{1}^{1}(t)} \sin \delta_{1}^{1}(t),
$$

где $\delta_{1}^{1}(t)$ - фаза $\pi \pi$-рассеяния в состоянии $I=J=1$. Результаты наших расчетов фазы $\pi \pi$-рассеяния приведены на рис. 3. Видно, что построенный нами формфактор дает достаточно близкое к результатам расчетов по формуле Брейта-Вигнера описание фазы рассеяния.

Как уже говорилось, фитирование по формуле Брейта-Вигнера хорошо описывает экспериментальные данные по формфактору пиона. Задача более точного описания фазы и характера резонансного поведения формфактора пиона в рамках нашего подхода связана с задачей выбора более реалистичной по сравнению с (16) волновой функции взаимодействующих кварков. Результаты расчетов сильно зависят от такого выбора, что позволяет надеяться на возможность более точного описания эксперимента.

\section{6. ЗАКЛЮЧЕНИЕ}

В рамках развитого авторами варианта релятивистской составной кварковой модели построено аналитическое продолжение электромагнитного формфактора пиона из области пространственноподобных переданных импульсов в комплексную плоскость. Показано, что в комплексной плоскости значений квадратов переданных импульсов формфактор пиона в релятивистской конституентной модели обладает такими же аналитическими свойствами, как и в подходе КТП. Полученный 
формфактор пиона во времениподобной области имеет резонансное поведение. Построенное аналитическое продолжение формфактора удовлетворяет дисперсионному соотношению и условию унитарности. Сказанное составляет основной результат настоящей работы: показано, что нет радикальных отличий в аналитических свойствах формфактора пиона, полученных в рамках РКМ и в КТП. Кроме того, полученные результаты дают разумное описание экспериментальных данных.

\section{Список литературы}

[1] P. A. M. Dirac, Rev. Modern Phys., 21:3 (1949), 392-399.

[2] B. D. Keister, W. N. Polyzou, "Relativistic Hamiltonian dynamics in nuclear and particle physics", Advances in Nuclear Physics, v. 20, Plenum Press, New-York, London, 1991, $225-479$.

[3] E. Gilman, F. Gross, J. Phys. G, 28:4 (2002), R37-R116, arXiv: nucl-th/0111015.

[4] G. R. Farrar, D. R. Jackson, Phys. Rev. Lett., 43:4 (1979), 246-249.

[5] G. P. Lepage, S. J. Brodsky, Phys. Lett. B, 87:4 (1979), 359-365.

[6] A. V. Efremov, A. V. Radyushkin, Phys. Lett. B, 94:2 (1980), 245-250.

[7] G. M. Huber, H. P. Blok, T. Horn et al. [The Jefferson Lab. $F_{\pi}$ Collaboration], Phys. Rev. C, 78:4 (2008), 045203, 16 pp.

[8] A. V. Radyushkin, Nucl. Phys. A, 532:1-2 (1991), 141-154.

[9] V. Braguta, W. Lucha, D. Melikhov, Phys. Lett. B, 661:5 (2008), 354-259, arXiv: 0710.5461.

[10] S. R. Amendolia, M. Arik, B. Badelek et al., Nucl. Phys. B, 277 (1986), 168-196; S. R. Amendolia, B. Badelek, G. Batignani et al., Phys. Lett. B, 146:1-2 (1984), 116-120.

[11] F. Gross, Relativistic Quantum Mechanics and Field Theory, John Wiley \& Sons, New York, 1999.

[12] А. В. Радюшкин, ЭЧАЯ, 20:1 (1989), 97-154.

[13] H. A. Bethe, E. E. Salpeter, Phys. Rev., 82:2 (1951), 309-310.

[14] M. Gell-Mann, F. E. Low, Phys. Rev., 84:2 (1951), 350-354.

[15] A. A. Logunov, A. N. Tavkhelidze, Nuovo Cimento. Ser. 10, $29: 2$ (1963), 380-399.

[16] D. Ebert, R. N. Faustov, V. O. Galkin, Eur. Phys. J. C, 47:3 (2006), 745-755, arXiv: hep-ph/0511029.

[17] J. F. Donoghue, E. S. Na, Phys. Rev. D, 56:11 (1997), 7073-7076, arXiv: hep-ph/9611418.

[18] W.W. Buck, R.F. Lebed, Phys. Rev. D, 58:5 (1998), 056001, 12 pp., arXiv: hep-ph/9802369.

[19] B. V. Geshkenbein, Phys. Rev. D, 61:3 (2000), 033009, 7 pp., arXiv: hep-ph/9806418.

[20] D. Melikhov, O. Nachtmann, V. Nikonov, T. Paulus, Eur. Phys. J. C, 34:3 (2004), 345-360, arXiv: hep-ph/0311213.

[21] M. Belička, S. Dubnička, A. Z. Dubničková, A. Liptaj, Phys. Rev. C, 83:2 (2011), 028201, 4 pp., arXiv: 1102.3122.

[22] B. Ananthanarayan, I. Caprini, I. S. Imsong, Phys. Rev. D, 85:9 (2012), 096006, 10 pp., arXiv: 1203.5398.

[23] H. R. Grigoryan, A. V. Radyushkin, Phys. Rev. D, 76:11 (2007), 115007, 12 pp., arXiv: 0709.0500.

[24] H. R. Grigoryan, A. V. Radyushkin, Phys. Rev. D, 76:9 (2007), 095007, 8 pp., arXiv: 0706.1543.

[25] S. J. Brodsky, G.F. de Téramond, Phys. Rev. D, 77:5 (2008), 056007, 20 pp., arXiv: 0707.3859.

[26] H. J. Kwee, R. F. Lebed, JHEP, 01 (2008), 027, 17 pp., arXiv: 0708.4054.

[27] С. Н. Соколов, ТМФ, 36:2 (1978), 193-207. 
[28] H. Leutwyler, J. Stern, Ann. Phys., 112:1 (1978), 94-164.

[29] F. Coester, Progr. Part. Nucl. Phys., 29 (1992), 1-32.

[30] W. N. Polyzou, Ch. Elster, W. Glöckle, J. Golak, Y. Huang, H. Kamada, R. Skibiński, H. Witala, Few-Body Systems, 49:1-4 (2011), 129-147.

[31] B. Bakamjan, L. H. Thomas, Phys. Rev., 92:5 (1953), 1300-1310.

[32] W. N. Polyzou, Phys. Rev. C, 82:6 (2010), 064001, 9 pp., arXiv: 1008.5222.

[33] В. Е. Троицкий, Ю. М. Широков, ТМФ, 1:2 (1969), 213-221.

[34] A. F. Krutov, V.E. Troitsky, Eur. Phys. J. C, 20:1 (2001), 71-76, arXiv: hep-ph/9811318.

[35] A. F. Krutov, V. E. Troitsky, N. A. Tsirova, Phys. Rev. C, 80:5 (2009), 055210, 5 pp., arXiv: 0910.3604.

[36] А.Ф. Крутов, В.Е. Троицкий, ТМФ, 116:2 (1998), 215-224.

[37] A.F. Krutov, V.E. Troitsky, Phys. Rev. C, 65:4 (2002), 045501, 17 pp., arXiv: hep-ph/0101327.

[38] А. А. Чешков, Ю. М. Широков, ЖЭТФ, 44 (1963), 1982-1992.

[39] A.F. Krutov, V.E. Troitsky, Phys. Rev. C, 68:1 (2003), 018501, 4 pp., arXiv: hep-ph/0210046.

[40] V.A. Matveev, R. M. Muradyan, A. N. Tavkhelidze, Lett. Nuovo Cimento. Ser. 2, 7:15 (1973), 719-723.

[41] S. J. Brodsky, G. R. Farrar, Phys. Rev. Lett., 31:18 (1973), 1153-1156.

[42] V. A. Matveev, L. A. Slepchenko, A. N. Tavkhelidze, Phys. Lett. B, 100:1 (1981), 75-78.

[43] P. L. Chung, F. Coester, B. D. Keister, W. N. Polyzou, Phys. Rev. C, 37:5 (1988), 2000-2015.

[44] P. L. Chung, F. Coester, W. N. Polyzou, Phys. Lett. B, 205:4 (1988), 545-548.

[45] W. Jaus, Phys. Rev. D., 44:9 (1991), 2851-2859.

[46] F. Schlumpf, Phys. Rev. D, 50:11 (1994), 6895-6898, arXiv: hep-ph/9406267.

[47] F. Cardarelli, I. L. Grach, I. M. Narodetskii, E. Pace, G. Salmè, S. Simula, Phys. Rev. D, 53:1 (1996), 6682-6685, arXiv: nucl-th/9507038.

[48] C. Bruch, A. Khodjamirian, J.H. Kühn, Eur. Phys. J. C, 39:1 (2005), 41-54, arXiv: hep-ph/0409080.

[49] Д. В. Ширков, В. В. Серебряков, В. А. Мещеряков, Дисперсионные теории сильных взаимодействий при низких энергиях, Наука, М., 1967.

Поступила в редакцию 24.08.2012, после доработки 11.10.2012 